\title{
Influence of B1 code correlation loop for vector tracking structures under complicated environment
}

\author{
WANG Qian ${ }^{1, *}$, SHANG Feng ${ }^{1}$, DU Liming ${ }^{2}$, and ZHOU Wenjia ${ }^{1}$ \\ 1. School of Electronic Engineering, Xi' an University of Posts and Telecommunications, Xi' an 710121, China; \\ 2. Qingdao Huatong Intelligent Research Institute, Qingdao 266111, China
}

\begin{abstract}
The code tracking loop is a key component for user positioning. The pseudorange information of BeiDou B1 signals has been fused and changed for vector tracking, so a correlation output model for complex scenarios is designed to prevent the propagation of error and valuate the signal performance. The relevant software and hardware factors that affect the output are analyzed. A single channel time-division multiplexing (TDM) method for multicorrelation data extraction is proposed. Statistical characteristics of the correlation output data for both vector and scalar structures are evaluated. Simulation results show that correlation outputs for both structures follow normal or Chi-squared distributions in normal conditions, and the Gamma distribution in harsh conditions. It is shown that a tracking model based on the multi-channel fusion hardly changes the probability distribution of the correlation output in the normal case, but it reduces the ranging error of the code loop, and hence the tracking ability of the code loop for weak signals is improved. Furthermore, vector tracking changes the pseudorange characteristics of channels anytime, and affects the mutual correlation outputs of the code loops in the abnormal case. This study provides a basis for the subsequent design of autonomous integrity algorithms for vector tracking.
\end{abstract}

Keywords: vector tracking, signal quality, code correlation, pseudorange error, scalar tracking, correlation peak loss.

DOI: $10.21629 / J S E E .2019 .06 .01$

\section{Introduction}

Since the BeiDou navigation satellite system (BNSS) was launched in 2012, it has been widely used in many civil and military fields. Due to the applications of the BNSS in various economic sectors, the precision and reliability of the navigation system have become a concern. Thus the evaluation system performance has been a focus by the assessment of navigation [1,2], signal quality [3], and kinematic positioning [4]. The signals on the main frequency of navigation system, which are intended for civil use and

\footnotetext{
Manuscript received November 06, 2018.

*Corresponding author.

This work was supported by the National Natural Science Fundation of China (41474027).
}

labeled as B1 [5], have a symbol rate of 2.046 MCps and a period of $1 \mathrm{~ms}$. Compared with the signals intended for military use, they have a narrower bandwidth and a shorter pseudo random period, which makes them susceptible to multipath interference, spoofing, interference and other adverse factors, thus reducing positioning accuracy and signal quality.

In order to improve the precision and reliability in adverse conditions, two main methods have been proposed: vector tracking and wide and local area differential techniques [6]. The differential technology is used for improving positioning precision through the relevance of the priori information, which is unable to reduce the error caused by signal attenuation. Consequently, the vector tracking technology has attracted great attention from the industry due to its outstanding performance.

The concept of vector tracking was first proposed in [7]. Unlike the traditional channel tracking methods, which are applied independently and lack associations, vector tracking uses information fusion to integrate all tracking loops that participate in operations into a closed loop, wherein channels are assisted by each other. Therefore, in complicated application conditions, such as high sensitivity, antiinterference, high dynamics [8,9] and fast recovery after signal interruption, vector tracking achieves better performance than traditional receiver tracking methods [10].

However, through the fusion of information, vector tracking changes the original signal processing structure of a single loop [11], which causes errors among all channels, and thus the signal quality analysis becomes more difficult. Hence, it is important to investigate whether vector tracking allows the enhancement of the quality of the observed signals, because the users should be informed whether vector tracking eliminates errors and enhances capabilities of signal processing through signal monitoring or not.

Monitoring and analysis of pseudorange on scalar tracking have not been suitable for vector tracking from the 
above principle. A new monitoring method needs to be designed to evaluate signal quality for the vector tracking structure although it is simple and practical especially for the user receivers. Depending on the navigation signal composition, the observation of signal quality mainly involves three aspects: signal power monitoring, joint surveillance of the spreading code and carrier, and correlation peaks' monitoring [12]. Among them, correlation peak monitoring involves the entire process of signal capturing and tracking from user terminals, while it also allows the removal of the influence of other factors, so it directly reflects signal quality and accuracy [13]. At first the accuracy of the differential code bias can be attained by the correlator of the ground-based monitoring station [14]. The ground observation data are used to detect abnormal signal-in-space (SIS) [15].

The relation between the performance of the receiver and the signal parameter is studied by real BNSS data [16]. Motivated by the above research, in this paper, based on the concept and application of the correlation peak, unlike the previous ground station, the aim is to design a new timedivision multiplexing (TDM) method of signal analysis for the general vector tracking receivers.

The traditional analysis of pseudorange distribution is not suitable for vector tracking. A new way based on the correlator is designed at the low hardware cost for the difference of scalar and vector tracking. The practical way of analysis is provided by the distribution, carrier-to-noise ratio (CNR) and symmetry. Moreover, the distribution of the correlator output cannot be changed by other channels in the normal condition, but it can be affected by fault propagation of channels in the extreme case.

The paper is organized as follows. In Section 2, the related research on the correlation output model in normal environments is presented. In Section 3, a data extraction scheme based on the multi-correlator and quality assessment algorithm is explained. The proposed scheme is validated by simulations and obtained results are presented in Section 4. Moreover, real data are used in experiments in order to verify the proposed scheme, and experimental results are provided in Section 5. Finally, a brief conclusion is given in Section 6.

\section{Mathematical model of correlation output}

\subsection{Signal model}

BeiDou signals usually consist of three parts: carrier, spreading code, which is also known as ranging code, and data message. The receiver ranging function is realized through the correlation of the received broadband signal and a corresponding local replication. The pseudorange values, which are required for positioning, are assembled according to the code phase of the correlation output. The length of the BeiDou B1 signal ranging code is 2 046, and the intermediate frequency signal, which is composed of the I and Q components, is defined by

$$
\begin{aligned}
& S_{\mathrm{B} 1}(n)=A_{\mathrm{B} 1 \mathrm{I}} C_{\mathrm{B} 1 \mathrm{I}}(n) D_{\mathrm{B} 1 \mathrm{I}}(n) \cos \left(2 \pi f t+\varphi_{\mathrm{B} 1 \mathrm{I}}\right)+ \\
& A_{\mathrm{B} 1 \mathrm{Q}} C_{\mathrm{B} 1 \mathrm{Q}}(n) D_{\mathrm{B} 1 \mathrm{Q}}(n) \sin \left(2 \pi f t+\varphi_{\mathrm{B} 1 \mathrm{Q}}\right)+w
\end{aligned}
$$

where $A_{\mathrm{B} 1 \mathrm{I}}$ denotes the signal amplitude, $C_{\mathrm{B} 1 \mathrm{I}}$ denotes the spreading code, $D_{\mathrm{B} 1 \mathrm{I}}(n)$ denotes the data message, $f$ represents the frequency, and $\varphi_{\mathrm{B} 1 \mathrm{I}}$ represents the carrier initial phase. Furthermore, the Q branch is similar to the I branch, and $w$ represents the noise.

When there is no external interference, the B1 signal is processed by the dispreading and demodulation module. The I and Q output signals are defined by

$$
\left\{\begin{array}{l}
I=A_{\mathrm{B} 1 \mathrm{I}} D_{\mathrm{B} 1 \mathrm{I}}(n) R_{\mathrm{B} 1 \mathrm{I}}(\tau) \operatorname{sinc}\left(f_{d} T\right) \cos \theta+w_{\mathrm{B} 1 \mathrm{I}} \\
Q=A_{\mathrm{B} 1 \mathrm{Q}} D_{\mathrm{B} 1 \mathrm{Q}}(n) R_{\mathrm{B} 1 \mathrm{Q}}(\tau) \operatorname{sinc}\left(f_{d} T\right) \sin \theta+w_{\mathrm{B} 1 \mathrm{Q}}
\end{array}\right.
$$

where $\tau$ denotes the code phase difference, $f_{d}$ represents the frequency difference, $\theta$ stands for the phase difference, and $T$ is the coherent integration time. Note that $R_{\mathrm{B} 1 \mathrm{I}}(\tau)$ represents the unit correlation function, which is defined as

$$
R_{\mathrm{B} 1 \mathrm{I}}(\tau)=\left\{\begin{array}{l}
\tau+1, \quad-1 \leqslant \tau<0 \\
-\tau+1, \quad 0 \leqslant \tau<1 \\
0, \quad \text { otherwise }
\end{array} .\right.
$$

In real environments, the most common form of interference is the multipath interference. Multipath signals can be mathematically expressed as amplitude attenuation and code phase delay. Similarly, the deceptive signals can be considered as multipath signals in a broader sense. The superposition of interference signals onto useful signals will eventually cause distortion of the correlation function. For instance, if the interfered I component is considered, when the useful signal is locked, the phase difference $\tau$ and the frequency difference $f_{d}$ approach zero, and the correlation output defined by (2) becomes

$$
\begin{gathered}
I=A_{\mathrm{B} 1 \mathrm{I}} D_{\mathrm{B} 1 \mathrm{I}}(n) \cos \theta+w_{\mathrm{B} 1 \mathrm{I}}+ \\
A_{\mathrm{B} 1 \mathrm{I}}^{s} D_{\mathrm{B} 1 \mathrm{I}}^{s}(n) R_{\mathrm{B} 1 \mathrm{I}}\left(\tau^{s}\right) \operatorname{sinc}\left(f_{d}^{s} T\right) \cos \theta^{s}
\end{gathered}
$$

where the superscript " $s$ " refers to the interference signal.

Regardless of the form, multipath, intentional or unintentional interference will eventually attenuate useful signals and decrease the signal-to-noise ratio (SNR). The SNR loss [17] is defined by

$$
\left(C_{S} / N_{0}\right)_{\theta f f, d B}=-10 \lg \left[10^{-\frac{\left(C_{s} / N_{0}\right)_{d B}}{10}}+\frac{k^{2}}{Q R_{C}}\right]
$$

where $\left(C_{S} / N_{0}\right)_{\theta f f, d B}$ denotes the interfered CNR, $\left(C_{S} / N_{0}\right)_{d B}$ denotes the CNR without interference, $Q$ 
stands for the quality factor and $R_{C}$ represents the B1 signal code rate. However, SNR loss causes the correlation output distortion. Thus CNR is an important characteristic of monitoring in the following experiment.

\subsection{Characteristic of correlation function}

The ranging code $C(t)$ can be thought of as an equal probability, independent, random binary pulse signal. The correlation function $R_{B 1 I}(\tau)$ is a random variable related to the modulation mode and integration range and has a constant period. Thus, the characteristic of the correlation function is obtained by considering it as a steady-state stochastic process [18]. The correlation function is defined by

$$
\mathrm{E}[C(t) C(t-\tau)]=R_{\mathrm{B} 1 \mathrm{I}}(\tau)
$$

where E denotes the expectation operator. Using (6), two characteristics can be obtained to describe the signal quality.

The first one is symmetry. The phase difference of the received and local pseudo-random code is a time-varying parameter in the high dynamic condition. The symmetry axis of the correlation function varies with the dynamics. The phase difference of the two codes is zero in the steady state. The equation of symmetry for the first order dynamic is defined by

$$
\mathrm{E}\left[R_{\mathrm{B} 1 \mathrm{I}}(q)\right]=\mathrm{E}\left[R_{\mathrm{B} 1 \mathrm{I}}(M \delta-q)\right]
$$

where $\delta$ denotes the code offset and $M$ denotes the chip numbers of the integration interval. From (7), it can be seen that the symmetry axis of the correlation function is $q=M \delta / 2$. The signal dynamic has much impact on the symmetry of the correlation function.

The second one is the correlation peak loss. In order to eliminate the influence of the carrier phase tracking error on the correlation output, and taking into account the various factors, such as the sum of squares of the I and Q components defined by (2), the complete correlation peak output in the static condition is defined by

$$
S_{p}=a \sqrt{\frac{2 C / N_{0} T}{u}} R_{\mathrm{B} 1}(\tau) \operatorname{sinc}\left(f_{d} T\right)
$$

where $u$ represents the sampling correction factor, such that when the receiver sampling frequency is two or more times greater than the signal bandwidth B1, the numerical value of $u$ can be equal to one [19]; $a$ denotes the sampling quantization coefficient of the A/D converter in the receiver's front end. According to (8), the amplitude of the correlation output is related to the strength of the received signal $C / N_{0}$, the carrier tracking frequency error $f_{d}$, the hardware parameters of the radio frequency (RF) front end, etc. Using (8), correlation peak ratios (CPRs) under different $\mathrm{CNR}$ values are calculated and the results are shown in Fig. 1. In theory, the corresponding CPRs for signal strengths of $25 \mathrm{~dB}-\mathrm{Hz}, 30 \mathrm{~dB}-\mathrm{Hz}, 45 \mathrm{~dB}-\mathrm{Hz}$ are 0.2 , 0.36 and 1 , respectively.

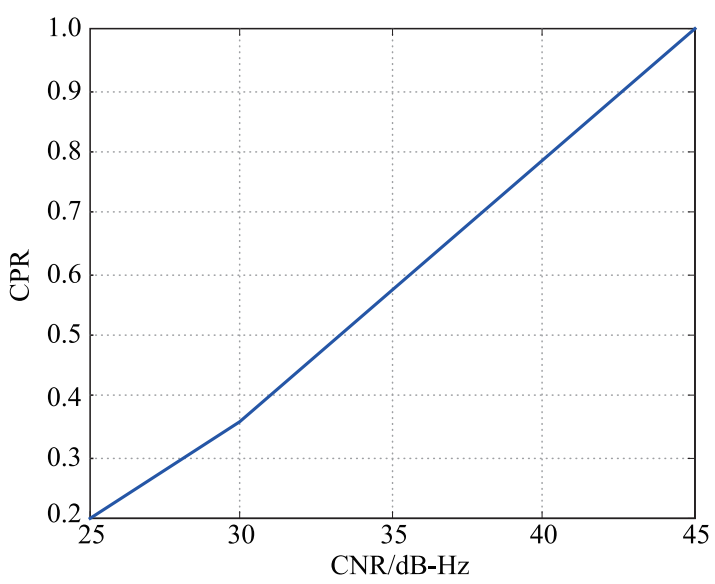

Fig. 1 CPR under different CNRs

\subsection{Comparative analysis of correlation model for vector and scalar tracking}

The block diagram of the correlation output with the corresponding vector and scalar structures is presented in Fig. 2. As it can be seen, the correlation output represents the matching between the locally generated code and the broadband code of the received navigation signal. The structural difference between the vector and scalar structures is based on the numerically controlled oscillator's (NCO) driving source. The NCO driver in the scalar structure is formed by single-channel filtered outputs, while the NCO driver in the vector structure represents the data output of the reverse NCO mapping [20] after multi-channel fusion in the position domain. The accuracy of the driver in the vector structure is much higher than that of the scalar structure in the pseudorange field.

The error variance of scalar loop equals

$$
\mathrm{E}\left\{\Delta \hat{\boldsymbol{\rho}} \Delta \widehat{\boldsymbol{\rho}}^{\mathrm{T}}\right\}=\boldsymbol{\sigma}_{v}^{2} \cdot \boldsymbol{I}_{m \times m}
$$

where $\boldsymbol{\sigma}_{v}^{2}$ is the matrix of measurement noise, $\boldsymbol{I}_{m \times m}$ is an $m$ dimensional unit matrix, $m$ is the number of visible satellites.

The error variance of vector loop equals

$$
\begin{gathered}
\mathrm{E}\left\{\Delta \widehat{\boldsymbol{\rho}} \Delta \widehat{\boldsymbol{\rho}}^{\mathrm{T}}\right\}=\boldsymbol{\sigma}_{v}^{2} \cdot \boldsymbol{H}_{m \times 4} \cdot\left(\boldsymbol{H}_{m \times 4}^{\mathrm{T}} \cdot \boldsymbol{H}_{m \times 4}\right)^{-1} \cdot \boldsymbol{H}_{m \times 4}^{\mathrm{T}}= \\
\boldsymbol{\sigma}_{v}^{2} \cdot \boldsymbol{W}_{m \times m} \\
\boldsymbol{H}_{m \times 4}=\left[\begin{array}{cccc}
a_{x, 1} & a_{y, 1} & a_{z, 1} & 1 \\
\vdots & \vdots & \vdots & \vdots \\
a_{x, m} & a_{y, m} & a_{z, m} & 1
\end{array}\right]
\end{gathered}
$$

where $a_{x, m}, a_{y, m}$ and $a_{z, m}$ are the components of the line of sight (LOS) vector pointing from the receiver to the number $m$ satellite. 


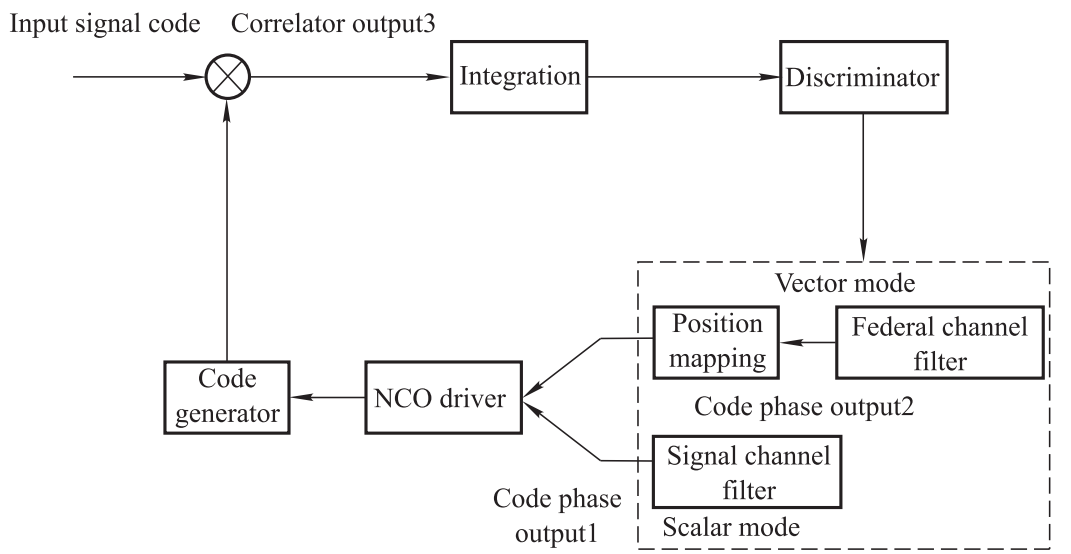

Fig. 2 Block diagram of the correlation output with the corresponding vector and scalar structures

Under the condition that the signal intensity of every channel is the same, the variance of the pseudorange noise is reduced from $\sigma_{v}^{2}$ in the scalar loop (Output1 of Fig. 2) to $\frac{4}{n} \sigma_{v}^{2}$ in the vector loop (Output2 of Fig. 2) according to the numerical analysis, where $n$ is the channel numbers which participate in the vector fusion [21]. Here the pseudorange error is equal to the code phase error discarding the additional system error.

By the above theory analysis, CNR is the main factor affecting the output of correlation (Output3 of Fig. 2). The equation of CNR change is as follows:

$$
\Delta d B=\frac{\Delta \tau}{L_{\mathrm{B} 1}}
$$

where $\Delta \tau$ is the square root of pseudorange error variance, $L_{\mathrm{B} 1}$ is the chip length of $\mathrm{B} 1$ signal codes. Table 1 shows pseudorange error and change of CNR on Channel 1 by vector tracking using data analysis. From Table 1, we can see owing to the big value of $L_{\mathrm{B} 1}(2046 \times 150)$ though vector tracking produces fewer pseudorange errors, the change of $\Delta d B$ is very insignificant. Thus vector tracking has far more impact on the pseudorange error than CNR. Besides, $\Delta \tau$ is related with the code offset in (7), so vector tracking changes the symmetry of the correlation function to a small extent contrasting to the scalar mode especially in the condition of a low CNR and high dynamics. The impact ratio between the thermal noise $\sigma_{t D L L}$ and the dynamic stress $R_{e}$ is 3 , which is explained by

$$
3 \sigma_{t D L L}+R_{e} \leqslant d
$$

where $\left[\begin{array}{ll}-d & d\end{array}\right]$ is the scope of discriminator pulling. While the loop bandwidth is $3 \mathrm{~Hz}$, the errors $R_{e}$ produced by dynamics stress of $10 \mathrm{~g}, 8 \mathrm{~g}$ and $6 \mathrm{~g}$ are $1.7532 \mathrm{~m}, 1.4025 \mathrm{~m}$ and $1.052 \mathrm{~m}$ respectively. Connected with the gain change $\Delta \tau$ attained by vector tracking in Table $1, R_{e}$ is in the same magnitude as $\Delta \tau$, thus vector tracking can reduce dynamics stress effectively.

Table 1 Pseudorange error and CNR change of channel-1 for vector tracking

\begin{tabular}{cccc}
\hline $\begin{array}{c}\text { Chl-1/ } \\
\text { dB-Hz }\end{array}$ & $\begin{array}{c}\text { Chl-[2:12]/ } \\
\text { dB-Hz }\end{array}$ & $\begin{array}{c}\Delta \tau \\
\text { Chl-1/m }\end{array}$ & $\begin{array}{c}\Delta d B \\
\text { Chl-1 }\end{array}$ \\
\hline 20 & 45 & 2.85 & $9.62 \mathrm{E}-6$ \\
25 & 45 & 2.15 & $7.24 \mathrm{E}-6$ \\
30 & 45 & 1.52 & $5.12 \mathrm{E}-6$ \\
35 & 45 & 1.32 & $4.44 \mathrm{E}-6$ \\
\hline
\end{tabular}

In theory, the correlator output is the basic parameter to test the signal performance for vector tracking. Because of the self-regulation function of the locked code loop, accuracy will gradually degenerate; once the lock is lost, the vector loop shows stronger signal tracking capability than the scalar loop.

\section{Data extraction based on multi-correlation}

Two commonly used methods for signal quality monitoring are the pseudorange differential method and the correlation value method. The pseudorange differential method requires all correlators to track the satellite signals to obtain the corresponding pseudorange, thus the required hardware cost is relatively high. While in the correlation value method, the input comes directly from the receiver front end, so it is more straightforward to implement comparing to the pseudorange differential method.

According to the above analysis in Section 2, pseudorange noise is the dominant factor determining the positioning error. The precision of pseudorange calculation mainly relies on the accuracy of signal synchronization, which ultimately depends on the correlation value of the instantaneous code during signal correlation. A simpler discrimination method is commonly used to test the symmetry of the leading code and the lagging code located on both sides of the instantaneous code. In non-ideal conditions, due to 
various types of interference, the shape of the signal correlation peak is not a standard triangle, which means that both sides of the instantaneous code might cause deviations. Consequently, more samples are needed to improve monitoring accuracy.

Since the monitoring receiver has sufficient hardware resources without the power and volume restrictions, the multi-correlation technique can be directly adopted. The multi-correlation technique is based on the fact that each receiver channel has a certain number of correlators, and all of them are able to provide different correlation values, which are then used to obtain the parameters of the satellite signal correlation function and to monitor the signal quality.

Due to the performance difference between ordinary and monitoring receivers, a time-for-space approach is generally adopted, which means that by using only one monitoring channel, the correlation peaks are sampled at different positions and at different times, and normalized by the correlation peak value. This correlation data extraction assumes that the dynamic properties do not change within a relatively short time period; otherwise the rapid change of Doppler will cause a relatively large sampling error.

In addition, due to the relatively small-scale and lowperformance hardware support in receivers, this method is more sensitive to processing factors, such as satellite elevation, antenna gain, and channel loss differences, so the correlation output at certain time moments is uncertain. Therefore, value averaging after normalization could eliminate the measurement errors, and even present the signal characteristics more accurately.

As can be seen in (3), when the code offset between the received signal and the local signal reaches one chip, the correlation value becomes zero. In general, the code offset in the monitoring channel can be set to $0-0.5$ chip. If we assume that $m$ is the satellite's serial number, of $f$ is the code offset, and $k$ is the sampling time, then the correlation output can be expressed as $J_{o f f}^{m}(k)$. With respect to the characteristics of the correlation output waveform, two important characteristics are defined: the distribution of the fixed code offset, and the joint distribution for different code offsets.

The multi-correlator structure for data extraction is presented in Fig. 3. Equipped with a high-speed clock, the correlator performs forward and backward correlation sampling with different time offsets using time slot division. Since the amount of data produced by the correlator on the complete integration interval is large, the structure samples the corresponding data around the specific clock tick to reduce the amount of calculations.

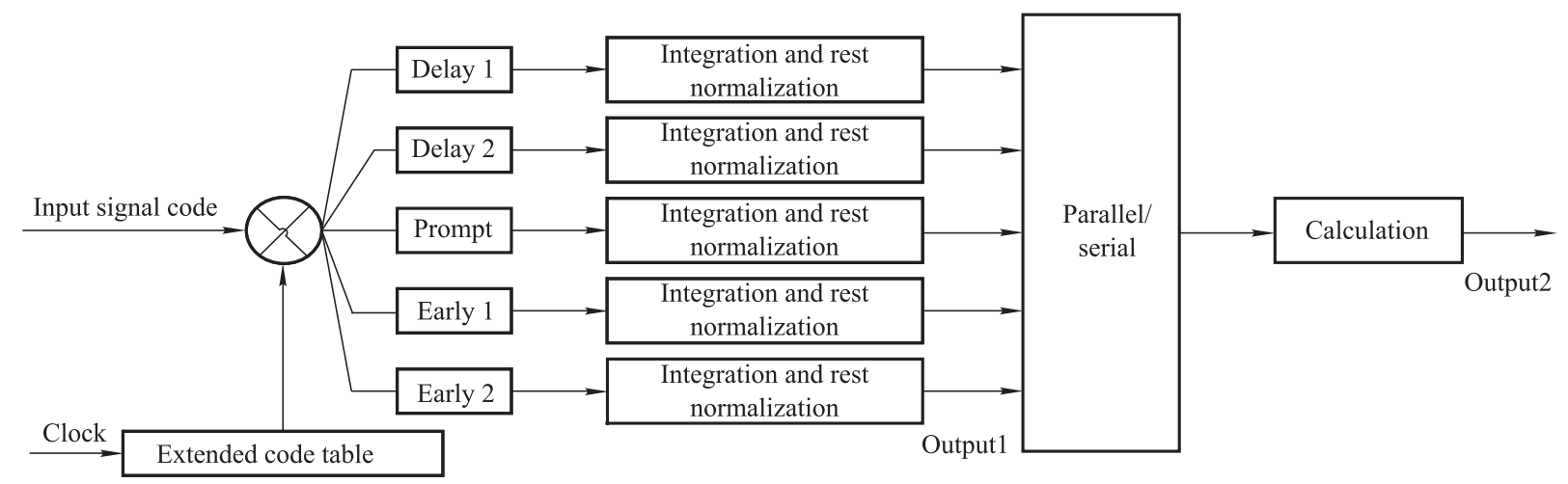

Fig. 3 Multi-correlator for data extraction

In order to eliminate the data vibration produced by noise, the data need to be smoothed by the filter.

The sampled data pass through the corresponding channels according to a predetermined classification. Then, they are normalized and loaded into storage areas. The statistical analysis on data in the storage area has shown that data distribution for the fixed code offset is strictly determined (Output1 of Fig. 3). Using serial-to-parallel circuit conversion, the joint distribution of data in the storage area for the case of different code offsets is obtained after data processing (Output2 of Fig. 3).

\section{Simulation and analysis}

\subsection{Scene setting}

According to the correlation output model presented in Section 2, we set the user receiver sampling rate to 16.368 MHz, the navigation signal strength CNR to the values from $25 \mathrm{~dB}-\mathrm{Hz}$ to $45 \mathrm{~dB}-\mathrm{Hz}$, the dynamic stress to the values from $0.1 \mathrm{~g}$ to $8 \mathrm{~g}$, the number of the signal tracking channels to 11 , the interval of coherent integration to $2 \mathrm{~ms}$, and the code offset to $\pm 0.5, \pm 0.25, \pm 0.125$, \pm 0.0625 and 0 chips. Both the vector and scalar loops use the extended Kalman filters with identical bandwidth parameters, which are shown in Table 2. 
Table 2 Bandwidth parameters setting of Kalman filters

\begin{tabular}{cccc}
\hline Symbol & Explanation & Number setting & Unit \\
\hline$S_{a}$ & Power spectral density (PSD) of processing noise for signal amplitude & $10 \mathrm{E}-3$ & $(\mathrm{~V} / \mathrm{s})^{2} / \mathrm{Hz}$ \\
$S_{c a r}$ & PSD of processing noise for signal carrier & $2 \cdot\left(4 \pi^{2} / 3\right) \cdot 10 \mathrm{E}-3$ & $(\mathrm{rad} / \mathrm{s})^{2} / \mathrm{Hz}$ \\
$S_{f}$ & Coefficient-1 of variance for Allan & $2 \mathrm{E}-19 \cdot c^{2} / 2$ & $(\mathrm{~m} / \mathrm{s})^{2} / \mathrm{Hz}$ \\
$S_{g}$ & Coefficient-2 of variance for Allan & $2 \mathrm{E}-20 \cdot c^{2} \cdot 2 \pi^{2}$ & $\left(\mathrm{~m} / \mathrm{s}^{2}\right)^{2} / \mathrm{Hz}$ \\
\hline
\end{tabular}

\subsection{Vector fusion analysis of different channels}

In order to explore the pseudorange effects of vector fusion among different channels, 11 channels are configured in the user receiver, whereby the signal strength of Channel 1 is $25 \mathrm{~dB}-\mathrm{Hz}$ and the signal strengths of the remaining channels are $45 \mathrm{~dB}-\mathrm{Hz}$. The code phase error distribution of Channel 1 and Channel 2 for both scalar and vector structures are evaluated.

From the simulation results, it can be concluded that regardless of the vector or the scalar channel, the signal parameter settings show no differences, but vector tracking reduces the tracking error of the code loop effectively. The tracking errors of Channel 1 and Channel 2 for the vector and scalar structures are presented in Fig. 4 and Fig. 5, respectively. The subfigure in Fig. 5 shows the code phase error of Channel 2 more clearly in a larger division drawing.

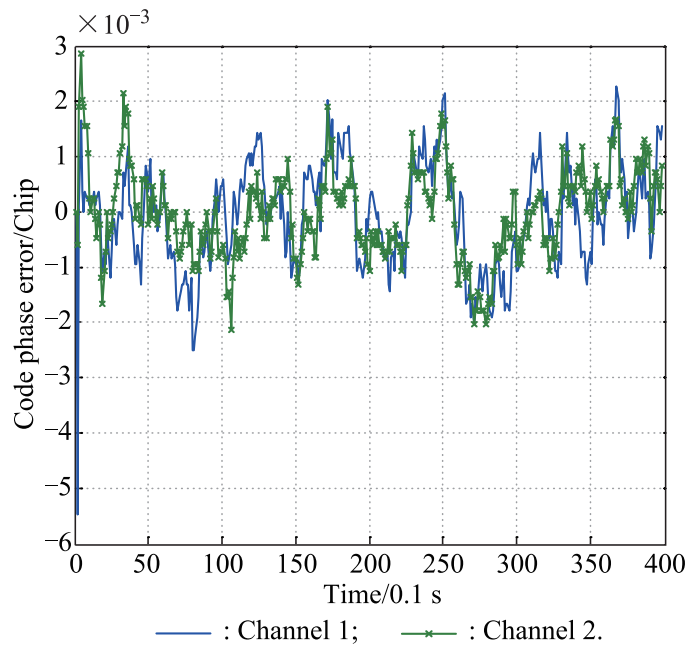

Fig. 4 Code phase error distribution of Channel 1 and Channel 2 for the vector structures

As can be seen from the figures, although the tracking errors of the strong channel for the two structures are at the same level, the code loop tracking error of the weak channel, assisted by the strong channel with the vector structure, is the same as the code loop tracking error of the strong channel (Fig. 4).

On the other hand, the weak channel shows a significant code loop tracking error with the scalar structure. Comparing the last two figures, it can be concluded that the mu- tual fusion among strong signal channels also provides a smaller ranging error than that of a single isolated strong signal channel, which fully proves the advantage of fusion at the information level in vector tracking. Code phase error (pseudorange error) can be used for scalar tracking and not for vector tracking to evaluate signal performance.

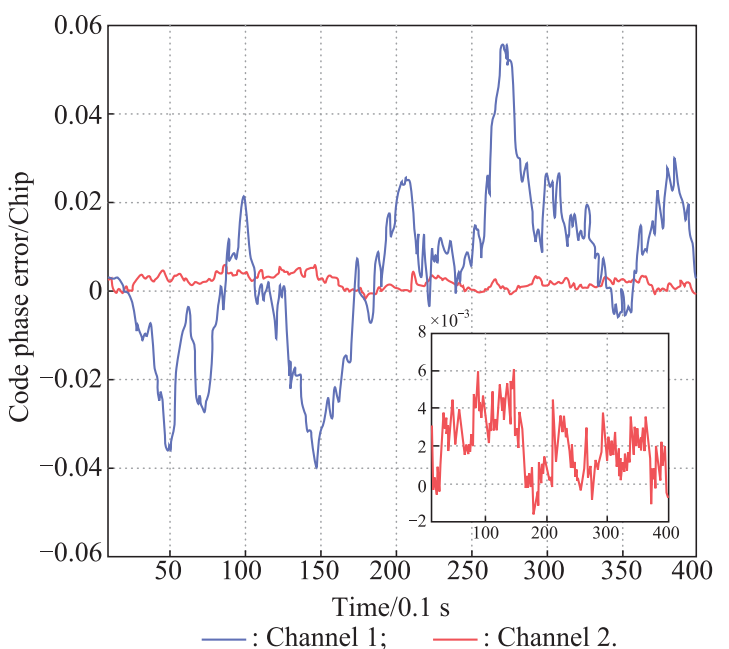

Fig. 5 Code phase error distribution of Channel 1 and Channel 2 for the scalar structures

Using the same experimental setting, Table 3 shows the symmetry distribution and CNR estimation of the weak and strong channels for the scalar and vector structures. These two parameters describe the quality of the signal. The process definition of symmetry is as follows:

$$
\text { Sym }=\frac{\mathrm{E}(\text { Amplitude average })}{\mathrm{V}(\text { Amplitude variance })} \text {. }
$$

Table 3 Symmetry distribution and CNR estimation of weak and strong channels

\begin{tabular}{|c|c|c|c|c|}
\hline \multicolumn{2}{|c|}{ Setting (CNR[1:11]/Dyn.) } & \multicolumn{3}{|c|}{ CNR[1]:25/CNR[2-11]:45/0.1 g } \\
\hline \multicolumn{2}{|c|}{ Code bias } & 0 & 0.125 & 0.25 \\
\hline \multirow{4}{*}{ Symmetry } & Scalar-Chl[1] & 3.4239 & 3.3034 & 3.2371 \\
\hline & Scalar-Chl[2] & 21.0620 & 18.5806 & 11.1829 \\
\hline & Vector-Chl[1] & 3.4010 & 3.2726 & 3.2601 \\
\hline & Vector-Chl[2] & 21.1195 & 18.7139 & 11.2689 \\
\hline \multirow{4}{*}{$\begin{array}{c}\text { CNR } \\
\text { estimation }\end{array}$} & Scalar-Chl[1] & \multicolumn{3}{|c|}{23.5232 (TSR:89.9\%) } \\
\hline & Vector-Chl[1] & \multicolumn{3}{|c|}{24.1194 (TSR:95.7\%) } \\
\hline & Scalar-Chl[2] & \multicolumn{3}{|c|}{44.8404} \\
\hline & Vector-Chl[2] & \multicolumn{3}{|c|}{44.9926} \\
\hline
\end{tabular}

From the table, although CNR and the tracking success ratio (TSR) of the weak channel are slightly increased by 
information of fusion for the vector structure contrasting to the scalar one, CNR gain of $0.6 \mathrm{~dB}$ can be decreased due to the limit of computational accuracy. In most cases the symmetry distribution and CNR estimation are consistent with the signal intensity irrelevant from the tracking structure. Thus, the features of correlation output are used for vector tracking to evaluate signal performance.

\subsection{Distribution for fixed code offset}

The probability distribution of the code stream for the vector structure without normalization when the signal strength is $45 \mathrm{~dB}-\mathrm{Hz}$ is presented in Fig. 6. When the code offset is equal to zero chip, the mean value is greater than that when the code offset is equal to 0.125 chip, which coincides with the output characteristic of code correlation.

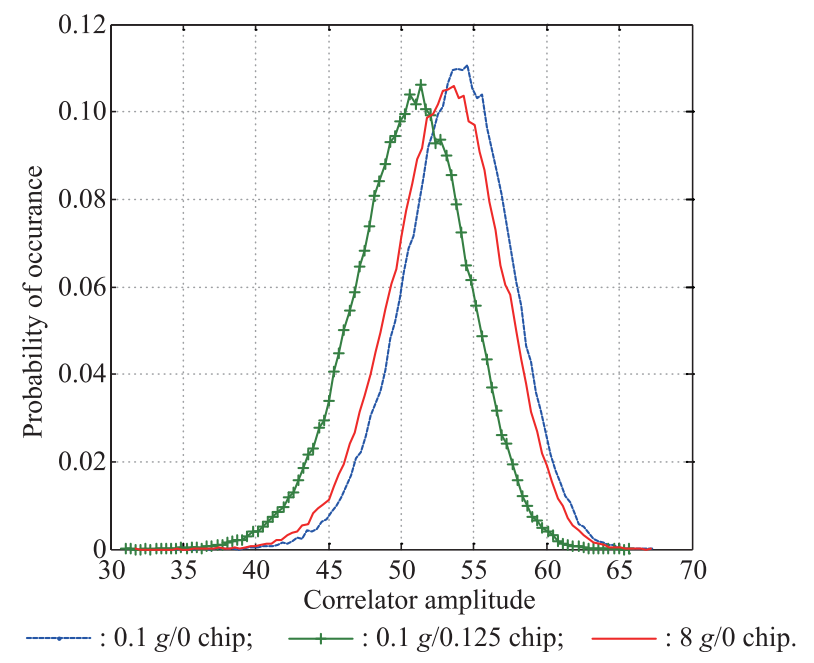

Fig. 6 Probability distribution of code stream for vector structure for different biases without normalization
The probability distribution of high dynamics is shown in the red curve. The rule of probability distribution is that the smaller the code bias, the bigger the correlator amplitude. Thus the code bias produced in the condition of high dynamics is in the scope of 0.125 chip, accordingly the red curve is in the middle of the blue and green ones. Regardless of the code offset value, the probability distribution basically coincides with the standard normal distribution after normalization. The above normal distribution is decided by Gauss white noise characteristics in theory. The distribution for the scalar structure is the same as that of the vector structure except that the correlator amplitude is decreased slightly in the strong signal of $45 \mathrm{~dB}-\mathrm{Hz}$.

Four typical settings are designed for intensive study of the correlator output. Table 4 shows the symmetry distribution and CNR estimation of different setting conditions. Referring to the theory analysis of Section 2 in this paper, code bias is a key factor of the impact symmetry. Signal dynamics and intensity lead to the code bias. From Table 3 signal intensity has far more impact on symmetry than signal dynamics. So is the impact analysis of the CNR estimation. Vector tracking slightly increases the value of CNR and symmetry compared to scalar tracking, which shows the merit of information fusion and the robustness of the vector loop especially in the condition of high dynamics and weak signals. In the condition of high dynamics, the symmetry of vector tracking is about 1 unit bigger than that of scalar tracking, which verifies that vector tracking reduces the dynamics stress effectively. However, the main character of correlation output is not changed by vector tracking.

Table 4 Symmetry distribution and CNR estimation of different setting conditions

\begin{tabular}{|c|c|c|c|c|c|c|c|}
\hline \multirow{2}{*}{\multicolumn{2}{|c|}{$\frac{\text { CNR[1:11]/Dyn. }}{\text { Code bias }}$}} & \multicolumn{3}{|c|}{$45 / 8 g$} & \multicolumn{3}{|c|}{$45 / 0.1 \mathrm{~g}$} \\
\hline & & 0 & 0.125 & 0.25 & 0 & 0.125 & 0.25 \\
\hline \multirow{2}{*}{ Symmetry } & Scalar & 19.0513 & 16.8291 & 10.1823 & 21.0316 & 18.5465 & 11.1061 \\
\hline & Vector & 19.9897 & 17.7222 & 11.0215 & 21.0865 & 18.6919 & 11.2211 \\
\hline \multirow{2}{*}{$\begin{array}{c}\text { CNR } \\
\text { estimation }\end{array}$} & Scalar & & 44.3039 & & & 44.8136 & \\
\hline & Vector & & 44.6501 & & & 44.9782 & \\
\hline \multicolumn{2}{|c|}{ CNR[1:11]/Dyn. } & \multicolumn{3}{|c|}{$35 / 4 g$} & \multicolumn{3}{|c|}{$30 / 0.1 \mathrm{~g}$} \\
\hline \multicolumn{2}{|c|}{ Code bias } & 0 & 0.125 & 0.25 & 0 & 0.125 & 0.25 \\
\hline \multirow{2}{*}{ Symmetry } & Scalar & 6.4072 & 5.7471 & 4.2768 & 4.5472 & 4.2842 & 3.7373 \\
\hline & Vector & 6.9058 & 6.3016 & 4.4700 & 4.5476 & 4.2919 & 3.7153 \\
\hline \multirow{2}{*}{$\begin{array}{c}\text { CNR } \\
\text { estimation }\end{array}$} & Scalar & \multicolumn{3}{|c|}{34.1914} & \multicolumn{3}{|c|}{29.5011} \\
\hline & Vector & \multicolumn{3}{|c|}{34.5873} & \multicolumn{3}{|c|}{29.9486} \\
\hline
\end{tabular}

When the signal strength is $25 \mathrm{~dB}-\mathrm{Hz}$ and the chip offset is set to zero, the loop is still in the locked state, but the amplitude distribution of the correlation output shows very large deviation compared to the standard normal distribution, as shown in Fig. 7. Namely, there is a significant rise at the central point of the vertical axis, and a relatively obvious tail between the 3 rd and the 5 th points on the $x$ axis. Moreover, the distribution of the quadratic sum of data in the three channels does not conform to the theoretical Chi-squared distribution (see the red curve in Fig. 8). 
According to the statistical theorem that the Chi-squared distribution is a special case of the Gamma distribution, the Chi-squared function (3) is converted to the Gamma $(1.5,2)$ function to match the actual distribution, and the interval values searching strategy is used to determinate both parameters of the Gamma function.

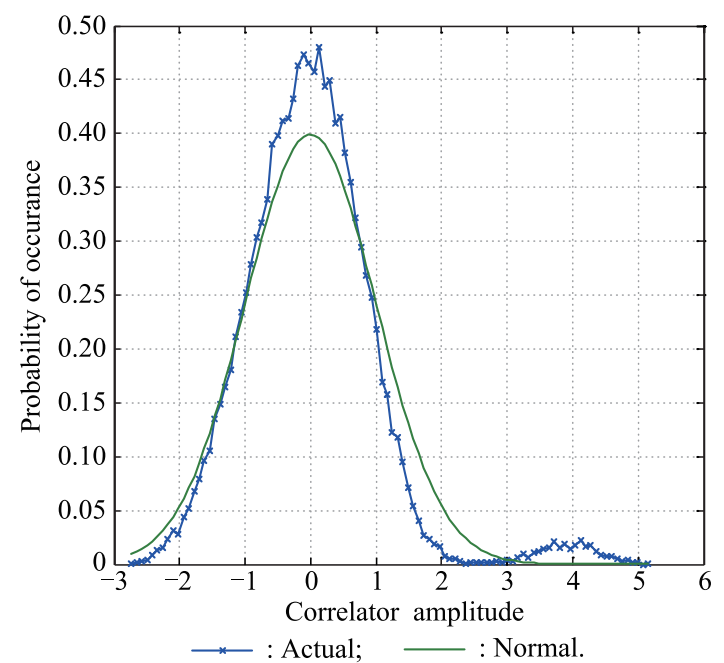

Fig. 7 Correlation amplitude distribution with weak signals

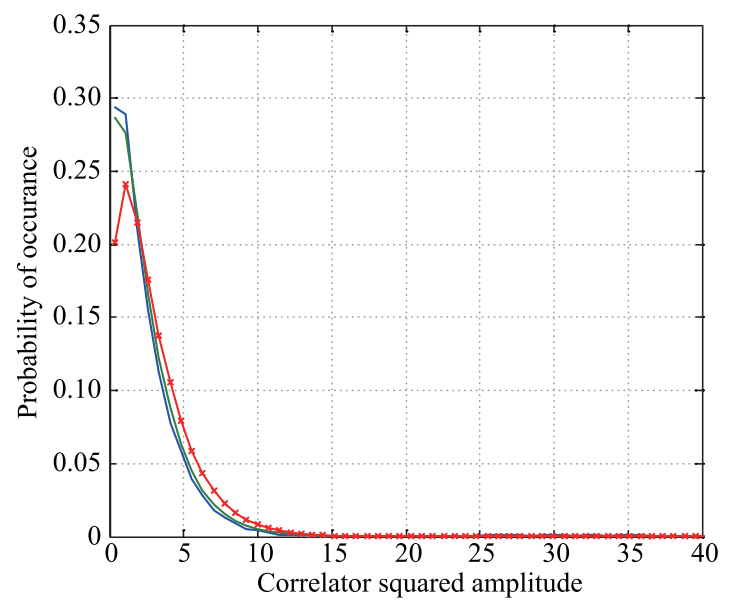

- $\operatorname{Gamma}(1.32,1.9) ; \quad \longrightarrow$ : Actual; $\quad$ «: Chi-squared(3)

Fig. 8 Correlation amplitude distribution of multi-channel with weak signal

The search problem is mathematically equivalent to parameter estimation, and the Kolmogorov-Smirnoff (K-S) distance [22] is used to test its estimation effect. The cumulative distribution function of the generalized Gamma distribution is listed, and parameter estimation is carried out according to the relationship between the sample points and their corresponding probability. Fig. 9 shows the change curve of the K-S distance in the calculation process. The final form of Gamma, with parameters $(1.32,1.9)$, is presented as a blue curve in Fig. 8. The above approach can be generalized and applied to detect abnormal signals, i.e., those which occur with abnormal probability.

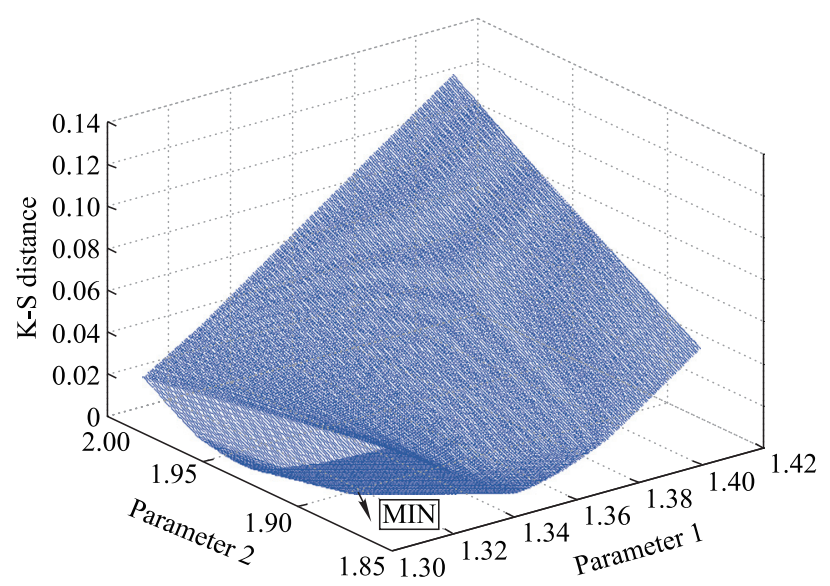

Fig. 9 Three-dimensional change curve of the K-S distance

\subsection{Correlator amplitude for different code offsets}

As specified in Section 4.1, nine different code offsets are used. In addition, using the multi-correlation joint extraction method presented in Section 3, the superimposed observations of the nine correlation channels are extracted and normalized, and nine discrete points are achieved, which are later used to obtain the actual correlation output curve as a mean value of the quadratic curve. The distribution curves, $S(x)$, at $25 \mathrm{~dB}-\mathrm{Hz}, 30 \mathrm{~dB}-\mathrm{Hz}$ and $45 \mathrm{~dB}-\mathrm{Hz}$ for both the vector and scalar structures are presented in Fig. 10. For different signal intensity values, the two sides of the curves are symmetrical around the null point of code bias on the condition that no multipath interference occurs.

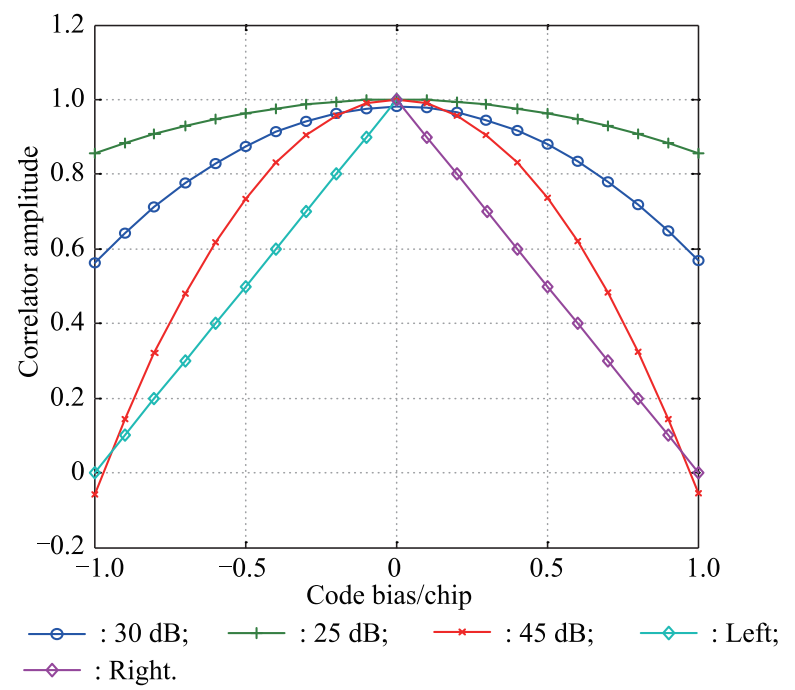

Fig. 10 Distribution curves at $25 \mathrm{~dB}-\mathrm{Hz}, 30 \mathrm{~dB}-\mathrm{Hz}$ and $45 \mathrm{~dB}-\mathrm{Hz}$ for both vector and scalar structures

The curve characteristic can be described by the peak slope value, which is defined by 


$$
G(x)=\frac{|S(0)-S(-1)|-|S(0)-S(1)|}{2} .
$$

As can be seen in Fig. 10, the numerical values of $G(45)$, $G(30)$ and $G(25)$ are $1,0.4$ and 0.16 , respectively, which fit the theoretical values of Fig. 1. The greater the signal strength is, the closer to theoretical value of one the peak slope is, which is consistent with the mathematical model described in the Section 2. This actually shows a new method of evaluating CNR by the curve graphic without complex computation.

\section{Experiment with real data}

\subsection{Design of data acquisition and playback device}

A mobile vehicle is used for data acquisition and playback analysis in a real scene. A data acquisition antenna at B1 frequency and two data calibration antennas at GPS L1 frequency are mounted on the car's roof. The configurations of interior vehicle equipment and joint interface are composed of a high precision GPS receiver, an acquisition and playback device, the radio frequency channel, and the power supply. The high precision receiver acts as the dynamic reference, and is comprised of a NovAtel SPAN FSAS. It has a joint navigation function provided by deeply coupled GPS/INS, which uses the local area differential positioning. In a complex road scene with a short time of occlusion, the positioning precision of this device is up to the centimeter level. The performance parameters of this data acquisition device are as follows: the sampling frequency is $100 \mathrm{MS} / \mathrm{s}$, the sampling bandwidth is $50 \mathrm{MHz}$, and quantization bits are 8 . In addition, it is able to playback real scene signals with high-fidelity quality, with a maximum CNR error less than $0.8 \mathrm{~dB}$ [23].

\subsection{Experimental results and analysis}

Test data from Beijing Zhongguancun district are collected using the vehicle-mounted data acquisition equipment, and are dealt with by the TDM method based on the vector loop and the scalar loop. Although the distributions of the correlation outputs of two different loops are the same as mentioned in Section 4, there is a difference in the continuity of CNR numbers of the real data.

As shown in Fig. 11, the CNRs of PRN\#1 (Geostationary Earth Orbit of BeiDou system) satellite signals are correspondingly calculated using scalar and vector loops. The difference of CNRs measured using scalar and vector loops is $0.25 \mathrm{~dB}$, which validates the simulation result that the vector loop is unable to improve the signal strength. The area where the signals are collected is downtown in an area full of high-rising buildings, so the shielding of signals is serious. Consequently, the blue curve of the scalar loop has some breakpoints, and its tracking success ratio is $85.7 \%$, while the red curve of the vector loop has better continuity due to the vector loop's stronger adaptability to weak signals; signals of up to $15 \mathrm{~dB}-\mathrm{Hz}$ could be caught using the vector mode.

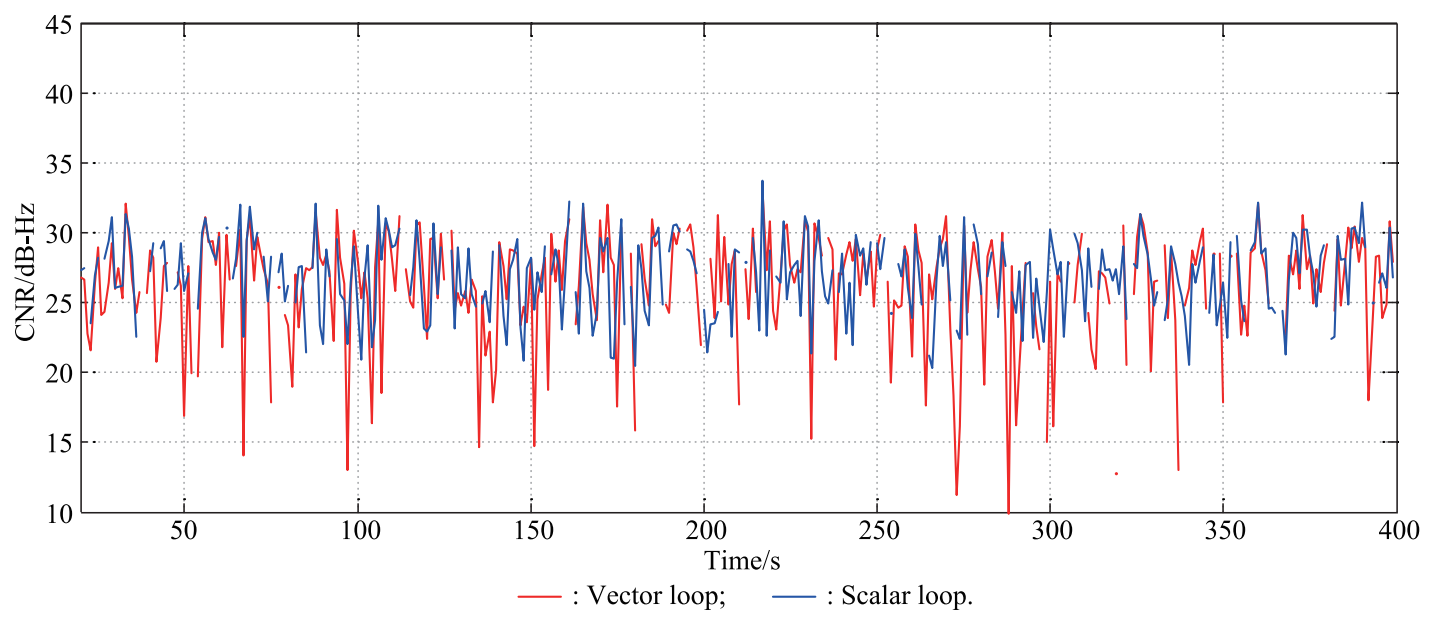

Fig. 11 CNRs of PRN\#1 satellite signals for scalar and vector loops

The situation of data acquisition outdoors is very complicated associating with the terrain, car speed, shelter and weather. Although in the case of the strong signal, the differences of CNR shown by scalar and vector loops are sometimes obvious. Fig. 12 and Fig. 13 show CNR com- parisons of two medium earth orbit (MEO) satellites signals processed by the vector and scalar loop structures. The frequency of the sampling point is $0.2 \mathrm{~Hz}$. CNR measured by the scalar loop is more vibrated than that of the vector loop by car acceleration in Fig. 12. CNR measured by the 
scalar loop is rapidly attenuated when the car encounters emergency brake compared with the vector loop in Fig. 13. These show the robustness of the vector loop in the complicated situation.

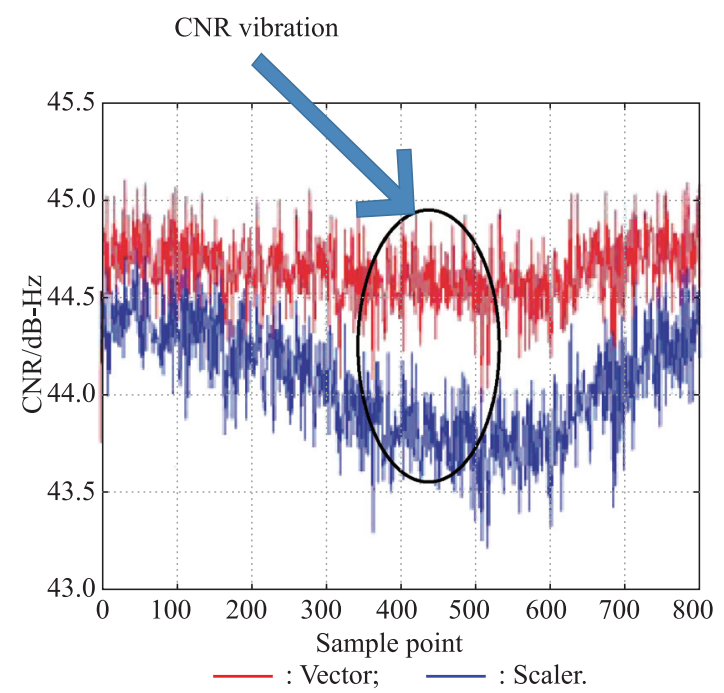

Fig. 12 CNR vibration of car acceleration

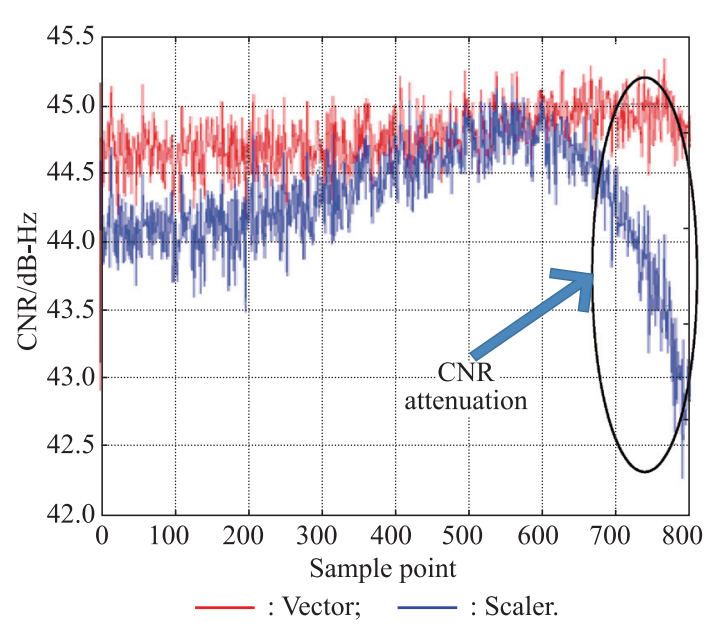

Fig. 13 CNR attenuation of emergency brake

Fig. 14 of the correlation output corresponding to CNR variety section in Fig. 12 and Fig. 13 is shown. From Fig. 14, the correlation amplitude is closely relevant to the number of CNR. The amplitude of the vector loop output is larger than that of the scalar loop output and so is the corresponding CNRs of the vector and scalar loops. This verifies the above conclusion that vector tracking really reduces dynamics stress effectively. This shows vector tracking affects the amplitude of the correlator output in the particular case.

To conduct an in-depth analysis the influence of complicated environments, special scenarios are set as follows: in the vector tracking channel architecture, abrupt pseudorange errors of $50 \mathrm{~m}, 100 \mathrm{~m}$ and $200 \mathrm{~m}$ are added to one of the signal channels respectively. The experiments show the maximum errors of correlation outputs of other signal channels because information fusions are $15 \mathrm{~m}, 25 \mathrm{~m}$ and $35 \mathrm{~m}$. In the above abnormal environment, the distribution of the correlator output is changed and anomaly detection algorithms need to be further studied.

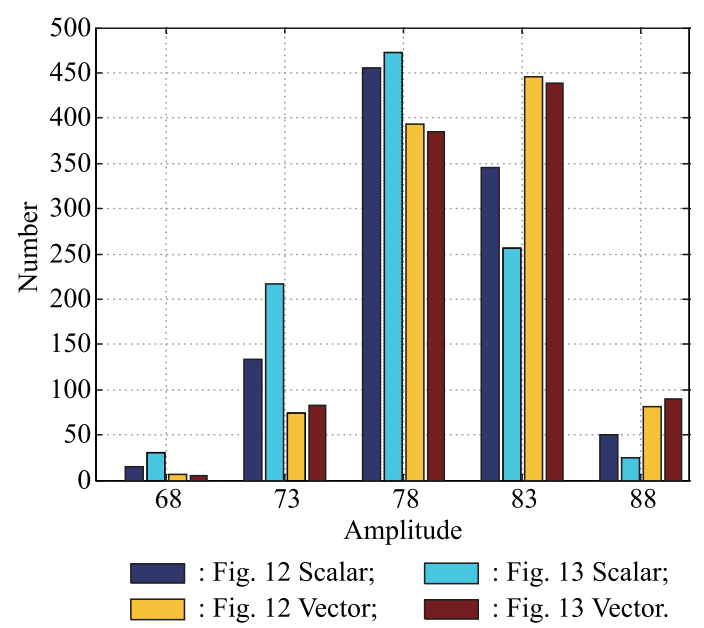

Fig. 14 Amplitude statistics of the scene in Fig. 12 and Fig. 13

\section{Discussion and conclusions}

This paper analyzes the code loop output of the BNSS B1-I branch for both scalar and vector tracking structures. The obtained analysis has shown the following: in normal conditions, the normalized code loop output has a standard normal distribution and superimposed multi-channel data follow a Chi-squared distribution, while in the case of weak signals the Chi-squared distribution degenerates to a Gamma distribution. Moreover, in the case where user hardware is certain, signal strength and dynamic are the dominant factors for the code loop output, while signal quality is measured by the gradient of the peak value.

In addition, it is shown that although the vector tracking structure slightly changes the symmetry and CNR, it is nearly unable to change the code loop output distribution and improve the signal strength by channel information fusion in the normal condition. Pseudorange distribution is priority selection for scalar tracking to test signals for the low design cost. Furthermore, it is proven that vector tracking can change data distribution at the pseudorange level substantially, and affect the correlator output between different channels in the abnormal case, so the output distribution of code correlation can be used for abnormal signal detection, and raising alarms; therefore, the reliability of the user terminal's service is improved. Future work needs to be done on how to forecast and find the fault of signals. 


\section{References}

[1] MONTENBRUCK O, HAUSCHILD A, STEIGENBERGER $\mathrm{P}$, et al. Initial assessment of the COMPASS/BeiDou-2 regional navigation satellite system. GPS Solution, 2013, 17(2): $211-222$.

[2] YANG Y X, LI J L, WANG A B, et al. Preliminary assessment of the navigation and positioning performance of BeiDou regional navigation satellite system. Science China Earth Sciences, 2014, 57(1): $144-152$. (in Chinese)

[3] DOU S H, KUANG C L, ZHOU Y Z, et al. Analysis of signal quality and navigation performance for Beidou system. Proc. of the 8th China Satellite Navigation Conference, 2017, 5: $671-681$.

[4] HE H B, LI J L, YANG Y X, et al. Performance assessment of single-and dual-frequency BeiDou/GPS single-epoch kinematic positioning. GPS Solution, 2014, 18(3): 393-403.

[5] LIU T H. Data research and analysis on B1 signal of BDS. Shanghai: Shanghai Jiao Tong University, 2013. (in Chinese)

[6] KIM S H, PARK K D. Improving DGPS accuracy by considering the correlation of pseudorange correction and satellite elevation angle. The Journal of Navigation, 2017, 70(6): $1267-1275$.

[7] SENNOTT J W. A flexible GPS software development system and timing analyzer for present and future microprocessors. Navigation, 1984, 31(2): 84-95.

[8] LIU J, CUI X W, LU M Q, et al. Vector tracking loops in GNSS receivers for dynamic weak signals. Journal of Systems Engineering and Electronics, 2013, 24(3): 349-364.

[9] ZHAO S H, LU M Q, FENG Z M. GNSS vector lock loop based on adaptive Kalman filter. Journal of Harbin Institute of Technology, 2012, 44(7): 139-143. (in Chinese)

[10] CHEN X Y, WANG X Y, XU Y. Performance enhancement for a GPS vector-tracking loop utilizing an adaptive iterated extended Kalman filter. Sensors, 2014, 14(12): 23630-23649.

[11] LASHLEY M, BEVLY D, PETOVELLO M G. What are vector tracking loops and what are their benefits and drawbacks? GNSS Solutions Column Inside GNSS, 2009, 4(3): 16-21.

[12] LIU D. Research on GPS signal monitoring technology. Beijing: Beihang University, 2012. (in Chinese)

[13] FENG X C, FU Y H, GONG L, et al. Detect technique and test analysis on navigation signal code correlation peak. Proc. of the 6th China Satellite Navigation Conference, 2015, 5: $517-$ 528.

[14] JIANG H, WANG H T, WANG Z M, et al. Real-time monitoring for BDS signal-in-space anomalies using ground observation data. Sensors, 2018, 18(6): 1- 17

[15] YANG J, YANG Y K, LI J S, et al. A novel satelliteequipped receiver for autonomous monitoring of GNSS navigation signal quality. Science China Technological Sciences, 2016, 59(7): $1137-1146$.

[16] ZHU L, ZHEN J, ZHAO X, et al. Real IF signal quality analysis using general GNSS software receiver. ZENG Q A. Eds. Wireless communications, networking and applications. New Delhi: Springer, 2016: 837-849.

[17] WANG J. Research on GNSS anti-spoofing technology by using acquisition module. Beijing, China: Tsinghua University, 2014. (in Chinese)
[18] LI C Q. The characteristics of PN code correlation and its applications under high dynamics. Changsha, China: National University of Defense Technology, 2005. (in Chinese)

[19] PANY T, MOON S W, IRSIGLER M, et al. Performance assessment of an under sampling SWC receiver for simulated high-bandwidth GPS/Galileo signals and real signals. Proc. of the International Technical Meeting of the Satellite Division of the Institute of Navigation, 2003: $103-116$.

[20] WANG Q, HU C B. Loop design of numerically controlled oscillator driven by multichannel united data. Journal of Chinese Inertial Technology, 2015, 23(4): 483-488. (in Chinese)

[21] WANG Q, CUI X, LIU J, et al. Quantitative analysis of the performance of vector tracking algorithms. High Technology Letters, 2017, 23(3): 238 - 244.

[22] CHANG K J. Optimality criteria methods using K-S function. Structural Optimization, 1992, 4: 213-217.

[23] XIAN D Y, FAN P R, WU H L. Test system for BDS user terminal based on RF replay apparatus. Proc. of the 7th China Satellite Navigation Conference, 2016, 5: 413-422.

\section{Biographies}

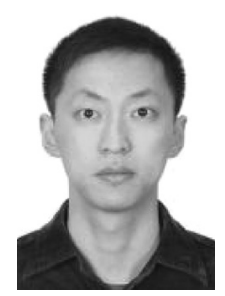

WANG Qian was born in 1978. He received his Ph.D. degree in Computer Science Department of Beihang University. He is a senior researcher in Xi'an University of Posts and Telecommunications. His research interests include the design of algorithms for signal tracking, integrated navigation and precision positioning.

E-mail: wqaloha@139.com

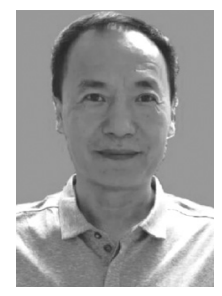

SHANG Feng was born in 1966. He received his M.S. degree in electromagnetic field and microwave technology of Xidian University. He is a professor in Xi'an University of Posts and Telecommunications. His research interests include the antenna theory and engineering, and radio frequency signal processing. E-mail: shangfeng@xupt.edu.cn

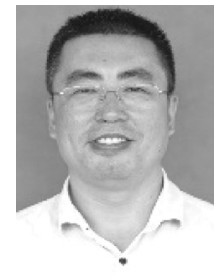

DU Liming was born in 1978. He received his M.S. degree in communication and information system of Nanjing University of Aeronautics and Astronautics. He is a senior engineer at Qingdao Huatong Intelligent Research Institute. He is mainly engaged in the research of digital signal processing and satellites navigation.

E-mail: xinde130@163.com

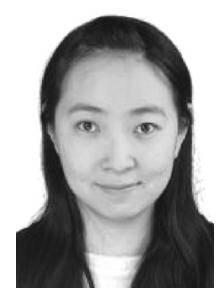

ZHOU Wenjia was born in 1986. She received her Ph.D. degree in control science and engineering, Northwestern Polytechnical University. She is a lecturer in Xi' an University of Posts and Telecommunications. Her research interests include microwave delay technology and microwave devices.

E-mail: zhouwj1986@163.com 